\title{
Evidencias exitosas de estrategias de gestión socioeconómica en el desarrollo económico sostenible y rentable en agro-industrialización de palma aceitera
}

\author{
Oscar Alejandro Tuesta Hidalgo \\ oath@hotmail.com \\ Escuela de Posgrado \\ Universidad César Vallejo
}

\section{RESUMEN}

La presente revisión ha tenido como objetivo: analizar la publicación de artículos originales de investigación descriptiva que tengan similitud acerca de evidencias de estrategias de gestión socioeconómicas que hayan conllevado al desarrollo económico sostenible y rentable derivado de la agroindustria de palma aceitera, ejerciendo una discusión crítica a las conclusiones. La metodología ha consistido en seleccionar 05 artículos acerca de una o las dos variables: estrategias de gestión socioeconómicas y desarrollo económico sostenible y rentable derivado de la agroindustria de palma aceitera. Entiéndase, que las estrategias de gestión socioeconómica subyacen en la producción de aceites y derivados. En ese tenor, el 2014 la producción alcanzó 200 millones de toneladas y la palma aceitera aportó $33 \%$ del total mundial. Asia aportó 52,8 \%, América 23,2 \% y Europa $19 \%$. Actualmente en el mundo existen dos grandes modelos de producción de palma aceitera: A. Grandes plantaciones conducidas por empresas transnacionales: Shin Yang, Nestle, Marks \& Spencer's \& General Mills, etcétera; y, B. El cultivo desplegado por pequeños productores. Muchas veces se presentan casos de modelos de combinación en los que funciona la colaboración entre pequeñas y grandes empresas. Se estima que el 2050 la población mundial alcanzará 9.100 millones de habitantes, convirtiéndose la demanda alimenticia en un factor decisivo para la sostenibilidad humana. En ese escenario, la agroindustria de palma de aceite y derivados resulta crucial para el desarrollo económico y sustentable de sus productores. Abundan críticas, pero la agroindustria de palma aceitera es una oportunidad para el desarrollo económico sostenible y rentable. No obstante, tiene que superarse los impactos ambientales negativos relacionados a tala de bosques, uso de suelos y todo daño irreversible a la biodiversidad, particularmente en la 
Amazonía. Paralelamente, se debe contrarrestar la corrupción de funcionarios, la violación de los derechos humanos y el despojo de tierras.

Palabras clave: estrategia de gestión; palma aceitera; desarrollo económico; rentabilidad. 


\title{
Successful evidence of socioeconomic management strategies in sustainable and profitable economic development in agro- industrializationoil palm
}

\begin{abstract}
The objective of this review has been: to analyze the publication of original descriptive research articles that have similarities about the evidence of socioeconomic management strategies that have led to sustainable and profitable economic development derived from the oil palm agribusiness, exercising a discussion criticism of the conclusions. The methodology consisted of selecting 05 articles about one or both variables: socioeconomic management strategies and sustainable and profitable economic development derived from the oil palm agribusiness. It should be understood that socioeconomic management strategies underlie the production of oils and derivatives. Along these lines, in 2014 production reached 200 million tons and oil palm contributed 33\% of the world total. Asia contributed 52.8\%, America 23.2\% and Europe 19\%. There are currently two major oil palm production models in the world: A. Large plantations run by transnational companies: Shin Yang, Nestle, Marks \& Spen-cer's \& General Mills, etcetera; and, B. The cultivation deployed by small producers. Many times there are cases of combination models in which collaboration between small and large companies works. It is estimated that by 2050 the world population will reach 9.1 billion inhabitants, making food demand a decisive factor for human sustainability. In this scenario, the oil palm and its derivatives agribusiness is crucial for the economic and sustainable development of its producers. Criticisms abound, but the oil palm agribusiness is an opportunity for sustainable and profitable economic development. However, the negative environmental impacts related to logging, land use and all irreversible damage to biodiversity, particularly in the Amazon, have to be overcome. At the same time, the corruption of officials, the violation of human rights and the dispossession of land must be countered.
\end{abstract}

Keywords: management strategy; oil palm; economic development; profitability

Artículo recibido: 10 Agosto. 2021 Aceptado para publicación: 07. Setiembre. 2021

Correspondencia: oath@hotmail.com Conflictos de Interés: Ninguna que declarar 


\section{INTRODUCCIÓN}

La sistematicidad de la presente revisión se inicia paralela a la búsqueda de evidencias difundidas que versen, por una parte, de estrategias de gestión socioeconómicas y por otra de evidencias exitosas de desarrollo económico sostenible y rentable dimanadas de la agro-industria de palma aceitera. En ese tenor, el objetivo del artículo radica en: Analizar la publicación de artículos originales de investigación descriptiva que tengan similitud acerca de evidencias de estrategias de gestión socioeconómicas que hayan conllevado al desarrollo económico sostenible y rentable derivado de la agroindustria de palma aceitera, ejerciendo una discusión crítica a las conclusiones. Está claro, que aquí no interesa, la situación problemática del desarrollo económico sostenible y su rentabilidad agroindustrial, sino el éxito del desarrollo económico sostenible y rentable derivado de algunas estrategias u operaciones asociadas a la agricultura e industria de la palma aceitera. En ese tenor, es que la autoría de esta revisión se formula la interrogante siguiente: ¿Qué evidencias exitosas se conocen en el Perú y en el mundo acerca de estrategias de gestión socioeconómicas que hayan conllevado al desarrollo económico sostenible y rentable relacionadas con la agro-industria de palma aceitera?

Para los intereses de la autoría, se necesita conocer, por una parte, la sistematicidad de las estrategias de gestión socioeconómica: finalidad, estudio de mercado, estudio técnico del proceso de producción, tamaño. campo de acción o localización, análisis de requerimiento de recursos, organización y gestión, análisis de impacto ambiental, calendarización de ejecución, estudio financiero, análisis de sostenibilidad y táctica de marketing.

Por otra, se necesita conocer las evidencias sistematizadas del desarrollo económico sostenible y rentable dimanado de la agro industrialización de palma aceitera. Es decir, alianzas estratégicas, la capacidad de generar ingresos suficientes y oportunos, la capacidad de gestión, solidez y permanencia, capacidad para adecuación a cambios tecnológicos. Así mismo, se necesita conocer las evidencias de la rentabilidad en términos de valor actual neto (VAN) y tasa interna de retorno (TIR).

\section{METODOLOGÍA}

En esa dirección y en ese sentido, se ha seleccionado y revisado los siguientes cinco artículos originales: 
- "Sostenibilidad económica, social y ambiental de la producción de aceite de palma en Malasia. Discusión y tendencias del mercado", artículo publicado en Kuala Lumpur por la Asociación Malaya de Aceite de Palma y Vengeta Rao en la revista Palmas del año 2004, Volumen 25, $\mathrm{N}^{\circ}$ Especial, Tomo I.

- “La palmicultura colombiana: sostenibilidad económica, social y ambiental”, artículo publicado en Nariño por Aylin Patricia Pertuz Martínez y Álvaro Enrique Santamaría Escobar en la revista Tendencias de la Facultad de Ciencias Económicas y Administrativas de la Universidad de Nariño del año 2014, volumen XV, $\mathrm{N}^{\circ} 1$.

- "Cambio de uso de suelos por agricultura a gran escala en la Amazonía andina: el caso de la palma aceitera”, documento publicado en Lima por Juan Dammert el año 2014 y producido por encargo de la Unidad de Apoyo de la Iniciativa para la Conservación en la Amazonía Andina (ICAA) liderada por International Resources Group (IRG) y sus socios: Sociedad Peruana de Derecho Ambiental (SPDA), Corporación de Gestión y Derecho Ambiental (ECOLEX), Social Impact (SI), Patrimonio Natural (PN) y Conservation Strategy Fund (CSF).

- "La agroindustria de la palma de aceite en América", artículo publicado por González en la revista Palmas del año 2016, Volumen 37, $\mathrm{N}^{\circ}$ Especial, Tomo II.

- "Estrategias financieras para incrementar la rentabilidad. Caso: Empresa Indema Perú S.A.C.”, artículo publicado por Zurita, Pucutay, Córdova y León en la revista de investigación INNOVA del año 2019. Volumen 4, No.3.1 pp. 30-41

\section{RESULTADOS Y CONCLUSIONES}

\section{Primera evidencia}

Si de resultados se trata, acera del desarrollo económico sostenible y rentable en lo que se refiere a la palma aceitera, Vengeta Rao (2004) revela que:

A partir de la siembra comercial de unas pocas hectáreas en 1917, la industria palmera en Malasia se ha convertido en una multibillonaria industria de 3,7 millones de hectáreas que suple una cantidad significativa de la demanda mundial de aceites comestibles. También ha contribuido sustancialmente a los ingresos del país y provee empleos directos e indirectos a cerca de medio millón de personas. (p. 1). Malasia, país ubicado en el sureste asiático, es citado a menudo como una muestra mundial de agroindustria exitosa de palma aceitera. 
Sin embargo, al 2020 el Consejo del Aceite de Palma de Malasia (MPOC), Kalyana Sundram, dijo que la demanda de este aceite vegetal más utilizado en el mundo podría "tocar fondo" después de los impactos provocados por el COVID-19 y la posible recuperación lenta.

Al margen del impacto del COVID-19 en el horizonte, antes del 2020, los resultados según Vengeta Rao (2004) se concentra en las conclusiones siguientes:

- En términos de sostenibilidad económica, la industria palmera en Malasia debe encarar dos problemas y dos tendencias. El primer problema es que la industria debe lograr una ganancia en productividad mayor al descenso del precio del producto. El espectacular éxito de la industria en el último siglo se debió a la mayor productividad, pero los rendimientos y tasas de extracción de aceite se han estancado en las dos últimas décadas. Inversiones en mecanización, producción de mejores plantas a través de cultivo de tejidos, obtención de variedades enanas de alto rendimiento, agricultura de precisión y dominio de la biotecnología son cruciales para mantener la productividad por encima de los costos.

- El segundo problema, en el que la industria depende de negociaciones internacionales, se refiere a las distorsiones comerciales de los aceites comestibles, en particular debido a los subsidios agrícolas en la Unión Europea y Estados Unidos.

- Las dos tendencias son: i) Incrementar las economías de escala a través de la expansión, tanto doméstica (en Sabah) como internacional, especialmente en Indonesia; ii) Mayor integración vertical a través de downstreaming, especialmente adquisición de operaciones existentes. Downstreaming genera mayor atención a la calidad del producto.

- Desde el punto de vista social, la sostenibilidad de la industria palmera en Malasia depende de una organización de plantación con mano de obra extranjera adecuada. Fuera de algunas cooperativas independientes y programas del gobierno, no existen cooperativas privadas de gran escala. De hecho, aún estos dos sistemas copian la forma en que las plantaciones manejan sus cultivos. Si la sostenibilidad de la industria es mejor a través del sistema de plantaciones, entonces la industria es dependiente de un suministro continuo de trabajadores inmigrantes. Además, a diferencia de los primeros inmigrantes, ahora la mano de obra extranjera es integrada en su mayoría por hombres solteros. La legislación actual sobre infraestructura social debe ser 
revisada para tomar en cuenta este cambio fundamental. Fuera del incremento de la mecanización para reducir la dependencia de mano de obra y las repetidas solicitudes al gobierno para reducir los costos de la mano de obra extrajera, por ahora no existen otras tendencias en el área de sostenibilidad social.

- Los temas relacionados con la sostenibilidad ambiental están ahora en primer plano debido a que la rápida expansión de la palma de aceite ha estimulado a las ONG ambientales. La mayor preocupación de estas organizaciones es la pérdida de bosques y biodiversidad con la expansión de la palma de aceite, aunque la información disponible muestra que la expansión ha sido a expensas del caucho, cacao y bosques degradados. La industria debe divulgar más ampliamente esta información crucial. Una tendencia emergente en sostenibilidad ambiental es la cada vez más estricta legislación local en relación a efluentes y emisiones. La estricta aplicación por parte del departamento del Medio Ambiente ha mantenido la industria palmera en Malasia como una industria limpia a pesar de su tamaño.

- Un tema que requiere más investigación es la producción de metano del sistema de tratamiento de efluentes. Buenas políticas agrícolas como la aplicación balanceada de nutrientes y el uso de técnicas de manejo integrado de plagas han ayudado a mantener bajo control la contaminación y la lixiviación en la industria del aceite de palma. (pp. 105 y 106). Las intelecciones de las conclusiones descritas han dado lugar a otra alternativa: la certificación del Aceite de Palma Sostenible de Malasia, que requiere que se cumplan con las normas de protección ambiental y las de los derechos de los trabajadores a concretarse en el 2020-2021.

\section{Segunda evidencia}

Ahora, se citará otra evidencia exitosa sobre el desarrollo económico sostenible y rentable relacionado con la palma aceitera escrutada del estudio: "La palmicultura colombiana: sostenibilidad económica, social y ambiental”, publicado por Pertuz y Santamaría (2014) quienes refieren varias aristas entre las que están las que a la autoría de la presente revisión le interesa: alianzas estratégicas, la capacidad de generar ingresos suficientes y oportunos, la capacidad de gestión, solidez y permanencia, capacidad para adecuación a cambios tecnológicos. Así mismo, se necesita conocer las evidencias de la rentabilidad en términos de valor actual neto (VAN) y tasa interna de retorno (TIR). 
Acerca de las alianzas estratégicas, he aquí lo que esgrimen Pertuz y Santamaría (2014):

Según FEDEPALMA, la evaluación a 23 alianzas que conforman al modelo de Alianzas Productivas Estratégicas (relaciones formales entre productores del sector), muestra que hay una mayor facilidad para la financiación, asistencia técnica y mayor formalización de relaciones con el Estado. Factores por mejorar: el estado sanitario de los cultivos, el orden público, créditos bancarios entregados inoportunamente y los cambios repentinos de los precios internacionales. Según la entidad, tanto la empresa como los productores ganan. (Castiblanco; Hortúa, 2012: 17). Vidal y Villaroel (2011: 148-157), en estudio realizado en María La Baja (Bolívar), consideran que el sistema de alianza estratégica entre pequeños productores de palma de aceite y los grandes productores y/o extractores genera beneficios a ambas partes y permite el sostenimiento económico de todos los involucrados. (Citado por Pertuz y Santamaría p. 178). Precisamente, son los beneficios mutuos, la razón de una o más alianzas estratégicas, porque conllevan a la suma de sinergias combinadas de lo mejor de cada empresa u organización.

Entiéndase que una alianza estratégica es la asociación, convenio o acuerdo de cooperación o colaboración que se realiza entre dos o más empresas, con la finalidad de obtener beneficios comunes tales como aumentar la producción, reducir costos, mejorar la rentabilidad, conseguir más clientes, acceder a nuevos mercados y canales de distribución, transferir tecnología, etcétera.

En lo que, a capacidad de generar ingresos suficientes y oportunos, se necesita saber, queda elucidada en la cita de Pertuz y Santamaría (2014) que se anota:

Las grandes inversiones en los cultivos de palma tienen que estar soportadas en diversas variables como la capacidad económica para establecer la plantación y esperar tres (3) o cuatro (4) años para obtener su fruto; la posibilidad de sobrevivir a las fluctuaciones del mercado; la facilidad para obtener préstamos bancarios; la posibilidad de integrar las plantaciones con la industria extractora y refinadora...; el poder político para influenciar las decisiones gubernamentales; y la posibilidad de invertir en infraestructura para la distribución (Mingorance et al, 2004: 24, 25). (Citado por Pertuz y 
Santamaría, p. 177). Estas son situaciones, que tienen que preverse y planificarse, precisamente, en una estrategia de gestión socioeconómica sui géneris.

Los mayores ingresos se dan a partir del año seis (6) y para optimizar los costos fijos y ser competitivo, según FEDEPALMA, se requieren 5.000 has. en promedio (citado por Ocampo, 2009: 176,177). Para el año 2005 el costo de producción es de 330 US\$/t, en tanto que el de Indonesia apenas supera los 200 US\$/t y el de Malasia es de 280 US\$/t. En el año 2007, el costo de producción aumenta a 340 US\$/, a diferencia de Indonesia y Malasia que se reduce a 154 US\$/t y 216 US\$/t, respectivamente (Infante; Tobón, 2010: 46). (Citado por Pertuz y Santamaría, p. 177). Seis años de espera, mientras tanto las personas responsables de la administración de la producción tienen que planear y tomar decisiones plasmadas en una estrategia de gestión socioeconómica peculiar.

En verdad, es todo un desafío de sobrevivencia a las fluctuaciones de mercado de la palma aceitera, entre otros factores cardinales; sin embargo, en perspectiva prospectiva resulta factible, es por eso que Pertuz y Santamaría (2014) expresan:

Empero, es un cultivo altamente productivo por hectárea/año. En el mercado mundial su precio es bajo y estable comparado con otros aceites vegetales, haciéndolo altamente competitivo en este mercado y viable económicamente para el biodiesel (Avellaneda, 2010: 6). (Citado por Pertuz y Santamaría, p. 177). Existen otros factores que los productores tienen que prever y planificar: facilidad para obtener préstamos bancarios, posibilidad de integrar las plantaciones con la industria extractora y refinadora, acceso al poder político para influenciar en las decisiones gubernamentales, etcétera.

Respecto a la capacidad de gestión lo que expresan Pertuz y Santamaría (2014) es la siguiente:

Entre los años 1996 y 2006 la participación promedio de valor agregado de la producción de aceite de palma en el PIB colombiano es de $1.6 \%$ con un crecimiento anual de 3.5\%. En las exportaciones, los principales beneficios económicos se dan entre los comercializadores, transformadores y grandes productores, dejando por fuera a los pequeños cultivadores (Pérez, 2010: 5). Las perspectivas al 2020 pretenden multiplicar la producción a 3.5 millones, e incrementar la productividad a 5.5 toneladas, con una tasa de crecimiento 
del 8\% anual y un crecimiento de exportaciones de un 78\% (Sánchez, 2009:

3). (Citado por Pertuz y Santamaría, p. 177). Evidentemente, los pequeños productores no son tan favorecidos.

Ahora bien, es necesario conocer acerca de la solidez y permanencia, dimensión que se desprende de la variable desarrollo económico sostenible y rentable en agroindustrialización de palma aceitera, situación sobre la que Pertuz y Santamaría (2014) dicen: "El cultivo requiere de un alto nivel de inversión para su establecimiento, debido a la preparación y mantenimiento del terreno en los primeros años improductivos, y al montaje de la infraestructura necesaria, desde la explotación hasta la extracción del aceite.” (Martínez et al, 2005: 20). (Citado por Pertuz y Santamaría, p. 177). Nuevamente esta situación pasa por pensar en una estrategia de gestión socioeconómica que para los pequeños y medianos productores quizá no sea otra que las alianzas estratégicas.

La capacidad para adecuación a cambios tecnológicos, es otra dimensión que se desprende de la variable desarrollo económico sostenible y rentable en agroindustrialización de palma aceitera, sobre la cual Pertuz y Santamaría (2014) reseñan lo siguiente:

El cultivo de palma aceitera es exitoso en el país en la última década debido a una proyección estratégica internacional, que tiene como base un "sistema organizacional especializado", unido a políticas del Estado que le han sido favorables (López, 2000: 131). En este sentido, el cultivo ha recibido grandes recursos por parte del Ministerio de Agricultura, que expresa que el 49\% de la propiedad es de los mismos agricultores. En la región de los Montes de María, los campesinos señalan que las tierras, en su gran mayoría, pertenecen a una sola familia (Preti et al, 2010: 8). (Citado por Pertuz y Santamaría, p. 177). Lo que aquí se colige es que las nuevas tecnologías han sido asimiladas, tanto por los campesinos propietarios de las tierras de cultivo como por los dueños de las tierras de una sola familia; siendo así, la optimización de la gestión encausa, también, a una adecuada toma de decisiones.

No obstante, la adecuación a los cambios de la tecnología, existen problemas medulares que lindan con la propiedad de la tierra, los medios de producción y la plusvalía. Es por eso que Pertuz y Santamaría (2014) dicen: 
La sostenibilidad económica del cultivo de palma de aceite tiene visiones encontradas en lo que respecta al pequeño y mediano productor, aunque la mayoría de autores considera que los pequeños productores no disfrutan de los beneficios económicos. En el sector, los grandes empresarios inducen a los campesinos a asociarse en proyectos de monocultivo gracias al endeudamiento. En realidad, los primeros sólo desean obtener materia prima sin ninguna vinculación laboral (Carrere, 2001:53). La apuesta exportadora del gobierno, en la que se encuentra la palma de aceite, implica convertir a los campesinos en empresarios prósperos, pero esto se evapora porque los pequeños campesinos serán desplazados a los cinturones de miseria de las ciudades, y algunos pocos solo serán mano asalariada de nuevas empresas exportadoras con pésimos sistemas de contratación (Tobasura, 2009: 31). (Citado por Pertuz y Santamaría, p. 178). Definitivamente, hay intereses opuestos. Los propietarios de las empresas exportadoras difícilmente compartirán la ganancia con los pequeños productores quienes prácticamente sólo venden su fuerza de trabajo.

Un punto de vista diferente es el que considera que la actividad palmera aumenta el empleo y les da estabilidad económica a los palmeros. En regiones como Tumaco, es percibida, entre otros aspectos, como una fuente de ingresos para la mano de obra no calificada (Perfetti et al, 2003:112). En la experiencia de Sabana de Torres (Santander), se considera que el modelo asociativo es válido para los pequeños productores, aunque, para ello, es necesario el acompañamiento de Ongs, al igual que la capacitación en aspectos asociativos (Baldovino, 2011:94). (Citado por Pertuz y Santamaría, p. 178). En verdad, el interés no pasa por el aumento del empleo de los palmeros, sino de que se mantenga esa mano de obra barata. Tampoco la esperanza para los productores pequeños estriba en la capacitación asociativa vinculada a Ongs. Los pequeños productores pueden tener conocimiento acerca de la constitución de la asociación, pero lo que no tienen es capital para participar con acciones, títulos o valores financieros que le den derecho sobre la propiedad de la empresa. Lo común, es que mientras más acciones tiene una persona, mayor poder de decisión o impedimento tendrá sobre la economía de la empresa. 
Finalmente, en lo que se trata de rentabilidad en términos de valor actual neto (VAN) y tasa interna de retorno (TIR), Pertuz y Santamaría (2014) refieren lo siguiente:

En cuanto al rendimiento del aceite de palma, este presenta una reducción de $8.3 \%$ en el año 2012, al pasar de 3.54 a 3.25 ton/ha. La productividad en Malasia es $18 \%$ superior, por lo cual se deben hacer esfuerzos para cerrar esta brecha de productividad. En el año 2012 se presenta una caída abrupta de los precios producto de la caída de los precios internacionales, la desgravación de los aceites vegetales importados (Acuerdo de Libre Comercio) y la revaluación del peso colombiano frente al dólar (Fedepalma, 2012: 38,42). Es de anotar que, en fechas recientes, desde el 2007 se reducen los rendimientos por hectárea debido a enfermedades y plagas (Silva; Martínez, 2009: 98). (Citado por Pertuz y Santamaría, p. 177). Para entender en forma clara la productividad, se necesita que se entienda el valor actual neto (VAN).

El valor actual neto (VAN) es un indicador financiero que sirve para determinar la viabilidad de un proyecto. Si tras medir los flujos de los futuros ingresos y egresos y descontar la inversión inicial queda alguna ganancia, el proyecto resulta viable.

Ahora bien, la producción de aceite de palma en Colombia no es competitiva si se compara con los países líderes que presentan costos de producción más bajos; pero los mayores costos de producción se trasladan al consumidor final "reduciendo su bienestar" (Cortés et al, 2012: 95). Según Finagro (2012), aunque el rendimiento promedio de Colombia en el año 2009 es de 3.4 ton/ha, el cual es inferior a Malasia e Indonesia, es superior al promedio mundial de 2.99 ton/ha (citado por García; Calderón, 2012: 75). (Citado por Pertuz y Santamaría, p. 177). Definitivamente, la competitividad colombiana con las empresas de Malasia e Indolencia es casi imposible.

La Federación Nacional de Cultivadores de Palma de Aceite de Colombia no puede competir con las empresas de Indonesia que pertenecen al Grupo Sinar Mas, al Grupo de Wilmar, a la Asociación de BGA (Bumitama Gunajaya Agro) junto al Grupo de IOI; al Grupo de Mas Musim y al Grupo de Lestari Astra, entre otras, Tampoco se puede competir con las empresas de Malasia: Shin Yang, Nestle, Marks \& Spencer's y General Mills.

Para INDUPALMA S.A., organización perteneciente a FEDEPALMA, el ejercicio económico desde al año 2006 es positivo, siendo sus mayores impactos la contratación 
laboral, el apoyo a proveedores, y a las Unidades Autónomas Empresariales, incluidas en las Cooperativas Asociativas de Trabajo (INDUPALMA, 2011: 38,39). (Citado por Pertuz y Santamaría, p. 178). En la actualidad, 20220-2021, la situación ha cambiado.

INDUPALMA S.A. es propiedad del grupo controlado por Daniel Haime Gutt y al 2020 enfrenta problemas con la clase trabajadora representada por el Sintraproaceites, complicaciones que lindan con la liquidación de la empresa.

\section{Tercera evidencia}

A continuación, se citará otra evidencia, pero ya no sobre el desarrollo económico sostenible y rentable relacionado con la palma aceitera, sino de un estudio comparativo de la situación y perspectivas del cambio de uso de suelos ocasionado por la instalación de monocultivos de palma aceitera en los bosques amazónicos de Colombia, Ecuador y Perú: "Cambio de uso de suelos por agricultura a gran escala en la Amazonía andina: el caso de la palma aceitera", publicación en la que se reseña una descripción del cultivo de la palma aceitera en esa región y discute los factores de su desarrollo, la conexión con procesos históricos de ocupación de la Amazonía, sus impactos sociales y ambientales (actuales y potenciales), así como los marcos regulatorios que intentan ordenar y prevenir la deforestación relacionada con la plantación indicada, tal como se relata en el documento de Dammert (2014):

En el caso de Colombia, la abundancia relativa de tierra fértil en otras zonas del país y la falta de seguridad asociada a la violencia política en los bosques amazónicos han disminuido la presión sobre ellos. Este escenario podría cambiar con una eventual pacificación del país y con la apuesta del gobierno por las "locomotoras de crecimiento" que implican la explotación de recursos naturales, en particular la minería. En la Amazonía ecuatoriana, por otra parte, el proceso de colonización se aceleró con los descubrimientos de petróleo a partir de la década de 1960. Las carreteras asociadas a la extracción petrolera facilitaron la expansión de la frontera agropecuaria. La Amazonía ecuatoriana, de menor tamaño que las de sus vecinos, es la que cuenta con una red de transporte en mejor estado y más extensa, en términos relativos. Por último, la Amazonía peruana es la más amplia y la más poblada, mayoritariamente con población colona. En el Perú, la región está atravesando por un boom de inversiones sin precedentes que podría cambiar 
irreversiblemente su situación política y social (Dourojeanni et al., 2009, citado por Dammert, 2014, p. 7). La percepción es que se trata de tres países de la Amazonia, pero que las zonas en las que se cultiva la palma aceitera hay oportunidades y debilidades distintas.

Al ocuparse de los impactos socio-ambientales, actuales y potenciales, Dammert (2014) expresa:

Los principales impactos sociales y ambientales de la palma aceitera en la Amazonía son, por un lado, las dinámicas de desposesión que afectan a los campesinos colonos y, por otro, la deforestación. La desposesión tiene que ver con el despojo de tierras, como ha ocurrido varias veces en Colombia, incluso con violencia paramilitar. En términos ambientales, existe una narrativa extendida, que amerita mayor investigación, sobre la posibilidad de establecer cultivos de palma aceitera en tierras ya deforestadas. En Ecuador y Colombia, la industria afirma que su expansión se produce principalmente reemplazando a otros cultivos o pastos para ganadería. En el caso peruano, en cambio, está documentado que los grandes monocultivos de palma instalados en los últimos tiempos lo han hecho largamente sobre bosques naturales. Desde un punto de vista ecológico, la palma aceitera requiere ciertas condiciones de precipitación, temperatura, gradiente y suelos. Este último factor es crítico. La palma puede reemplazar otros usos del suelo, pero siempre y cuando estos no lo hayan degradado al punto de que haya perdido sus nutrientes o esté acidificado. (p. 9). El sentido común de cualquier congénere, aunque no sea ambientalista, pasa por pensar con sensatez: en cualquier zona de la Amazonía que se tale bosque y se cultive palma aceitera habrá impactos ambientales negativos.

La palma aceitera mono-cultivada a gran escala implica, cambio del uso de los suelos de forestal a agrario y de grandes áreas de tierras. Sin embargo, en el mercado existe un déficit de producción de aceites vegetales y una de las alternativas pasa por la agroindustria de palma aceitera, entonces la recomendación es que se adopte una estrategia de gestión socioeconómica en la que se planifique con criterios técnicoambientalistas en toda la Amazonía. 
La conservación y protección ambiental pasa también la infracción de la legalidad y el fenómeno de la corrupción, tal como con claridad narra Dammert (2014):

Los marcos regulatorios en los tres países protegen a los bosques amazónicos a través de diversos mecanismos, como el establecimiento de áreas naturales protegidas, reservas forestales, bosques de producción permanente y tierras indígenas, además de distintos procedimientos que regulan el cambio de uso de los suelos. En los casos de Perú y Ecuador, por ejemplo, las empresas de palma requieren pasar por procesos de certificación ambiental para comenzar a operar. Sin embargo, existen varias ventanas para deforestar legalmente: la acreditación de la aptitud agrícola del suelo, la extracción de reservas forestales, la regularización de la propiedad informal deforestada, entre otras. Más aún, se evidencian prácticas de incumplimiento de la legislación, asociadas en parte a la debilidad de las organizaciones a cargo de que se la respete. En los tres países tenemos, además, procesos de descentralización de competencias relacionadas con el cambio de uso de los suelos, que si bien procuran acercar las decisiones a los territorios que se ven afectados por ellas, han sido objeto de críticas de corrupción y cooptación de gobiernos locales por ciertos poderes económicos. (p. 9). La Amazonía ha sido talada porque el aceite de palma es un negocio lucrativo. Siendo así, se presume la existencia de una conexión entre deforestación, comercio ilegal de tierras y corrupción de funcionarios.

He aquí, un párrafo que atañe a un pecado capital, según lo que dice OXFAN Internacional (2018):

El Grupo Romero, el actor económico más grande en el país, tiene ya 22.500 hectáreas de plantaciones de palma aceitera en funcionamiento y ha solicitado la asignación de más de 34.000 hectáreas adicionales de tierras públicas para palma aceitera. De ser aprobados, los cuatro nuevos proyectos planeados por el Grupo Romero generarán la deforestación de 25.005 hectáreas de bosques amazónicos (23.000 de las cuales son bosques primarios) en violación de la legislación peruana. (Párr. 6). Se entiende que, si se transgrede la ley emitiendo títulos de propiedad, se está frente a un hecho de corrupción de funcionarios. 
Como también, es de dominio público, desde hace tiempo, el "Grupo Melka" conducido por Denis Melka, empresario y dueño de un conglomerado de 25 empresas dedicadas a diversos rubros en el Perú, despliega un modelo de negocios que involucra la obtención de terrenos para la plantación de palma aceitera, hecho que en Malasia ya llevó a un impacto ambiental relacionado con la deforestación.

\section{Cuarta evidencia}

La prospectiva consustancial al desarrollo económico sostenible y rentable derivada se la agro industrialización de palma aceitera es la que se escruta del artículo "La agroindustria de la palma de aceite en América", publicado por González (2016):

Se estima que en el año 2050 la población mundial alcanzará 9.100 millones de habitantes, convirtiéndose la demanda por alimentos en un factor decisivo para la sostenibilidad de las personas, muchas de ellas en condición inferior a los niveles de alimentación adecuados. Es en este escenario donde el desarrollo de la palma de aceite y sus derivados desempeñan un papel importante para el desarrollo económico de las comunidades. (p. 216). Al margen de las críticas, la agroindustria de la palma aceitera es una oportunidad para el desarrollo económico y sostenible.

En esa lógica, la proyección en América, acorde con la Organización de las Naciones Unidas para la Alimentación y la Agricultura, muestra una tendencia de producción de alimentos creciente hacia el 2050, tal como se entiende en una cita más de González. (2016):

Teniendo en cuenta el crecimiento de la demanda de aceites y grasas per cápita, para el 2050 se requerirán 150 millones de toneladas adicionales de aceites y grasas vegetales. Para atender esa demanda se tendrían que sembrar “333 millones de hectáreas de soya o 217 millones de hectáreas de colza o 36 millones de hectáreas de palma de aceite"; esto en virtud de su alto rendimiento por unidad de superficie, y la versatilidad en sus usos tanto para la alimentación como para la industria, ya que la aparición de los biocombustibles puede cambiar algunas de las tendencias previstas $\mathrm{y}$ provocar un aumento de la demanda mundial, dependiendo principalmente de los precios de la energía y las políticas gubernamentales. (p. 217). La 
agroindustria, en términos de proyección, sustituye a la producción de hoja de coca asociada a crimen, deforestación, violencia social organizada y contaminación ambiental en la década de los 70 y 80, particularmente en los países amazónicos de Perú, Colombia y Bolivia.

La comparación de producción mundial de las principales grasas y aceites, entre los que provenientes de palma en el 2014 resulta considerable, razón por la que González (2016) expresa:

Respecto a la producción mundial de aceites y grasas, en 2014 fue de 200 millones de toneladas, de las cuales el aceite de palma aportó $33 \%$ del total mundial, siendo el aceite más utilizado para suplir la demanda mundial, seguido por el aceite de soya, con $22 \%$, y en tercer lugar el aceite de colza, con un aporte de $14 \%$. Asia fue el continente que realizó el mayor aporte, con 52,8 \%, seguido de América, con 23,2 \% y luego el continente europeo, con $19 \%$ (...) (p. 218). De la cita se colige, que la oportunidad es para los trabajadores que participan en la producción de palma aceitera y su consiguiente industrialización.

\section{Quinta evidencia}

Resultados relativos a una estrategia de gestión socioeconómica para el desarrollo económico sostenible y rentable derivada de la agro industrialización de palma aceitera, específicamente no se ha encontrado, salvo la publicación del estudio "Estrategias financieras para incrementar la rentabilidad. Caso: Empresa INDEMA Perú S.A.C.” de

\section{Zurita, Pucutay, Córdova y León (2019) quienes describen:}

Los factores que intervienen en los bajos niveles de rentabilidad son los elevados costos de producción, los cuales están relacionados con la mano de obra, materia prima y otros costos indirectos de fabricación, así también corresponde a los altos costos de los recursos humanos y tecnológicos. Para dar solución a la problemática se propone: Incrementar los ingresos, mediante estrategias de venta con el fin de captar de nuevos clientes, de la misma manera aplicar estrategias de marketing en base a las 4P: a) Producto, la calidad que busca todo emprendedor para ofrecer a sus consumidores, con la finalidad de satisfacer sus necesidades; b) Plaza, la captación de los nichos de mercado para comercializar los bienes; c) Precio, el valor final asignado a los 
productos comercializados en el mercado; d) Promoción, el punto de inicio para lograr afianzar los servicios con la intención de dar a conocer las ofertas al mercado competitivo. (Morelos y Nuñéz, 2017; Quinde y Ramos, 2018; Villegas, Hernández y Salazar, 2017; Ferré y Ferré, 1996; Kotler y Armstrong, 2003; Meoño y Escoto, 2006; Porter, 2005; Sallenave, 2002). (Citado por Zurita, Pucutay, Córdova y León, p. 32). Evidentemente, los factores que se presentan en las finanzas y en la economía oscilan entre favorables y desfavorables.

En ese orden de conceptos teórico-prácticos, la estrategia de gestión socioeconómica y el desarrollo económico sostenible y rentable, se entiende que son variables propias de la Economía, Administración, Contabilidad, entre otras ciencias afines. Por consiguiente, su praxiología como experiencia práctica posible se describe, explica y predice en las legalidades en términos de principios, leyes y reglas que configuran su teoría y su práctica de estos campos del conocimiento.

En la interpretación de los ratios financieras de rentabilidad según el análisis cuantitativo, se determinó que el ROA (Return on assets) disminuyó en los últimos tres años (en el año $2016,0.86 \%$, año $2017,0 \%$ y en el año $2018,0.36 \%$ ), por lo tanto, el rendimiento que generó la empresa producto de su inversión se redujo anualmente en un $0.40 \%$ aproximadamente.

Respecto al indicador ROE (Return on equity), los resultados disminuyeron a consecuencia de los altos costos de servicios, para el año 2016 fue de $2.25 \%$, en el año 2017 de $0 \%$ y para el año 2018 de $0.27 \%$, por ende, los resultados no fueron los deseados por los accionistas de la entidad.

Referente al nivel de ventas entre los periodos del 2016 al 2017 los resultados crecieron en un 175\%, mientras que en los periodo del 2017 al 2018 hubo una reducción de un 30\%, los mismos que fueron ocasionados por la inadecuada gestión del financiamiento, elevado costo de ventas, que en el periodo 2016 fue de 70\%, para el año 2017 de $110 \%$ y para el 2018 de $85 \%$, así como también la falta de planeación estratégica el cual se vio reflejado directamente en los resultados finales de los estados financieros.

En relación a los factores que influyen en la disminución de la rentabilidad como escases del apalancamiento, innecesarios costos de mano de obra, materiales y gastos indirectos, en el último año ha mejorado, sin embargo, este contexto requiere de un control firme, 
dado que existe una relación directa entre el manejo incorrecto de la producción y la administración para la toma de decisiones.

Para mejorar la rentabilidad de la empresa se elaboró estrategias financieras estableciendo los siguientes objetivos: Incrementar las ventas, reorganizar los costos de acuerdo a las necesidades por cada proyecto e incrementar la liquidez (...) (p. 33). En resumen, la cita de los autores trata de reducciones e incrementaciones, así como de las acciones que se recomienda para la superación de los factores inherentes a la gestión y al desarrollo económico sostenible y rentable.

\section{Discusión de las analogías}

Actualmente en el orbe terrestre existen dos grandes modelos de producción de palma aceitera. Por un lado, están las grandes plantaciones de palma aceitera, bajo la conducción de empresas privadas transnacionales tales como Shin Yang, Nestle, Marks \& Spencer's \& General Mills, etcétera. Por otro parte, el cultivo se desarrolla a través de pequeños productores. Diversas veces se da el caso de modelos intermedios donde se logra una combinación en la que funciona la colaboración entre los pequeños productores y las grandes empresas o alianzas estratégicas. En estos casos, la empresa privada se encarga de operar sus plantas procesadoras, con o sin una gran plantación propia, mientras que los pequeños productores se encargan de plantaciones a pequeña escala, y suministran la materia prima a las empresas transnacionales. En ese tenor global las estrategias empresariales expresan los fines de control y poder de grandes grupos económicos sobre empresas pequeñas, productoras y suministradoras de insumos a pequeña escala, situación que genera tensiones y relaciones discordantes como consecuencia de los intereses opuestos. Lo que ocurre, es que aquí se manifiesta las contradicciones antagónicas de dos clases sociales, propias del sistema capitalista neoliberal, que tienen intereses inmediatos a corto plazo e intereses estratégicos a largo plazo.

Las CLASES SOCIALES son grupos sociales antagónicos en que uno se apropia del trabajo del otro a causa del lugar diferente que ocupan en la estructura económica de un modo de producción determinado, lugar que está determinado fundamental-mente por la forma específica en que se relaciona con los medios de producción. (Harnecker, 1976, p. 114).Conviene precisar, que las contradicciones antagónicas son, ante todo, las existentes entre las clases, cuyos intereses tienen carácter inconciliable. 
De la revisión bibliográfica se aprecia y colige, que la agroindustria de palma aceitera o africana (Elaeis guineensis) es sostenible económicamente para las empresas transnacionales, dada la productividad y rentabilidad, aunque está muy distante a las de Indonesia y Malasia. Definitivamente, el cultivo de la palma aceitera exhibe una tendencia rentable y de crecimiento que justifican una expansión no sólo en el ámbito mundial, sino también en los países de la Amazonía Andina. La tendencia en mención se deriva de la demanda de aceites vegetales, tanto para la alimentación como para cosméticos o para que se use en biocombustibles. No obstante, el crecimiento de la palma no es igual en Colombia, Ecuador y Perú, países amazónicos a los que se les ha analizado. Así, los tres países están en momentos bastante diferentes en lo que respecta a su situación social y ambiental y a los debates públicos en torno a sus espacios amazónicos y su potencial de desarrollo. Las posibilidades de una expansión a gran escala de la agricultura capitalista son mayores, por ahora, en Perú, donde hay muchas tierras públicas y una política de fomento a las inversiones privadas facilitada en su concreción burocrática por algunos gobiernos sub nacionales ávidos de inversiones agrícolas a gran escala y por una falta de coordinación alarmante entre las instancias del gobierno nacional. En Ecuador y Colombia, la palma se expande principalmente en zonas que no son amazónicas, aunque hay un crecimiento visible y relativamente transparente en la Amazonía ecuatoriana y otro menos institucionalizado, por decirlo de alguna manera, en la colombiana. (Dammert, 2014, p. 65). Por ende, el desarrollo económico de la agroindustria de palma aceitera en la Amazonía Andina no constituye en la actualidad, un escenario que pudiera catalogarse en auge totalmente seguro. En los tres países andinos: Perú, Ecuador y Colombia, la situación es distinta.

Según, Villarán, F., Dávila, M., Urday, P., Sánchez, B. \& Vargas, R. (2014):

La cooperación internacional, a través del UNODC, indudablemente con una óptica acertada ha venido impulsando nuevos cultivos de Palma en el Pongo de Caynarachi en San Martin, en Pucallpa y Aguaytía, inclusive con modernas plantas extractora Neshuya y Alianza, cuya capacidad instalada atiende aún la oferta agrícola de las áreas adyacentes. (p. 62). En la cadena de palma aceitera, los principales actores son la empresa Palmas del Espino, la empresa asociativa INDUPALSA y la empresa asociativa OLPESA. Es decir, lo que se ve es un balance entre la empresa privada y las organizaciones de 
los pequeños productores. También es importante la participación de FREDEPALMA. Para el caso de la cadena del palmito, la producción se circunscribe a 2 empresas asociativas: ASLUSA y Alianza.

Siguiendo con Perú, según Leveau, R. (2018):

Las fincas productoras de palma aceitera en el valle del río Shanusi - Loreto, se caracterizan por ser gestionadas mayormente por hombres de un promedio de edad de 50 años, originarios de otra localidad, con estudios de primaria y secundaria, donde la mayoría (76 por ciento), tiene un área con cultivo de 5.46 ha. (p. 93). Evidentemente, estos datos revelan sostenibilidad y rentabilidad actual.

De acuerdo con Salinas, E., Mucho, E., Amado, E. \& Mendoza, J. (2017):

La palma aceitera es uno de los principales productos industriales para la producción de insumos como la cosmética, alimentaria y combustibles. Por ello, la palma aceitera producida en el Perú, hoy en día tiene un gran potencial por la diversidad de microclimas y espacios territoriales, los cuales se necesitan para poder cultivar y expandir este producto. Además, la industria de palma aceitera es beneficiada por diversos acuerdos comerciales y por ello también el gobierno ha puesto énfasis en la producción de palma aceitera en el Perú, considerándola en el Plan Estratégico Nacional hasta el año 2026. (p. 2). Los conceptos que aquí se esgrimen, evidencian también sostenibilidad y rentabilidad.

En el Perú oficial, uno de sus objetivos principales que se formula el Ministerio del Ambiente (2016), es el siguiente: "Promueve la gestión de emisiones de GEI de los productores de palma aceitera." (p.31). Se trata de producir, teniendo en cuenta las medidas de mitigación apropiadas para cada país encausadas a la disminución de emisiones de gases de efecto invernadero y a la conservación de sumideros de carbono que apoyan a los esfuerzos globales de la gestión de emisiones.

En Colombia, según la Comisión Económica para América Latina y el Caribe (2019):

La industria de aceite y biodiésel de palma ha crecido notablemente en la última década, con una fuerte expansión del cultivo que, en 2017, abarcaba más de 400000 ha, las cuales aportaban materia prima para 11 plantas procesadoras. Ese mismo año, esas plantas produjeron 513000 toneladas de 
biodiésel, destinado a cubrir la demanda interna y la exportación. La mayor parte de las plantas están en la zona norte del país. (p. 93). En Guatemala, Perú, República Dominicana y en el resto de países de Latinoamérica y el Caribe también se produce palma aceitera que alcanzan extensiones inmensas.

\section{En materia de sostenibilidad y rentabilidad, Quintero \& Torres (2019) describen:}

En el año 2010, Cenipalma realizó un estudio en las plantas de beneficio de las diferentes zonas palmeras de Colombia, concluyendo que del cuesco total obtenido el $67 \%$ se utiliza como combustible para calderas, $16 \%$ se dispone para la venta a otras industrias como combustible, 5\% es utilizado en compostaje, $7 \%$ como acondicionador en las vías de las plantaciones y $5 \%$ en otros usos. (p. 9). La producción de residuos de la palma aceitera evidencia una ventaja económica en sintonía con el medio ambiente.

$\mathrm{Al}$ referirse a la rentabilidad proveniente del cultivo de palma aceitera, PRODESIS en el 2005, citado por Linares, Zapata, Nazar \& Suáres (2018) se dice: “Actualmente las compañías industriales trasnacionales como Unilever, Nestlé, Procter \& Gamble, Kenkel, Cognis y Cargill dominan el mercado mundial de palma de aceite” (p. 2). Para las empresas transnacionales, definitivamente el cultivo de palma aceita es una actividad sumamente rentable, siendo está una razón para que se cuente con apoyo financiero de organismos multilaterales como el Banco Mundial, y el creciente mercado internacional. No obstante, la rentabilidad derivada del cultivo de palma aceitera, hay también discrepancias respecto a la sostenibilidad ambiental, es por ello que Villafuerte (2018), describe tal situación:

El Movimiento Mundial por los Bosques Tropicales considera que el aceite de palma es una de las industrias de rápido crecimiento y más integradas como agro- negocio, lo cual contrasta por ser de las menos estudiadas en términos de sus impactos sociales y ambientales. Esta organización estimó para Guatemala una superficie cultivada con palma de 130000 hectáreas, las cuales representan alrededor del 4 por ciento de la superficie agrícola del país, localizadas en los departamentos de Petén, Izabal, Alta Verapaz, Quiché, Escuintla, Suchitepéquez, Quetzaltenango, San Marcos y Retalhuleu, donde se han producido desplazamientos de comunidades indígenas 
o expulsiones en la idea de Saskia Sassen. Incluso, va más allá: “cuando una empresa o un gobierno extranjero adquiere una gran extensión de tierra para plantar palma para la producción de biocombustibles, expulsa floras y faunas enteras, pequeños agricultores, instalaciones manufactures rurales y mucho más” (p. 5). El caso descrito, es una manifestación de las principales contradicciones y consecuencias que devienen del cultivo de la palma aceitera en Guatemala.

La rentabilidad y lo sostenibilidad están asociadas, como es obvio, a mejorar la producción mediante la innovación de métodos, tal como la utilización de los residuos industriales para la variedad Deli x Nigeria en pre-viveros. Sobre esta experimentación se ocupan Madrigal, Á. \& Garbanzo, G. (2018) quienes reportan lo siguiente:

Encontramos que el tratamiento con $80 \%$ de suelo $+20 \%$ de EFB (S20F) aumentó la longitud y el diámetro de la hoja, la biomasa, el diámetro del bulbo y la longitud de la raíz. También aumentó la absorción de nutrientes en todas las mezclas. La concentración de $\mathrm{Mg}, \mathrm{K}, \mathrm{P}, \mathrm{Zn}$ y S fue mayor que en el suelo. Los desechos industriales permiten una reducción significativa en los fertilizantes para los pre-viveros. (p. 1). Qué duda cabe, la palma aceitera es un cultivo importante que necesita para una mejor producción de mejores técnicas, pericias, métodos etcétera.

Siendo el cultivo de palma aceitera una oportunidad en términos rentables y sostenibles, en el Ecuador el Ministerio de Agricultura, Ganadería, Acuacultura y Pesca ha creado consejos consultivos para que encausen la producción agrícola, tal como lo describe Naranjo (2016): "En diciembre de 1999 se creó el Consejo consultivo del banano. Posteriormente en junio de 2003 el Consejo consultivo de la palma aceitera grasas y aceites, y en abril de 2004 la codificación de la Ley especial del sector cafetalero" (p. 60). Lo que se hizo en el Ecuador, son políticas de comercialización interna, emanadas de las denominadas mesas de concertación o espacios de diálogo entre el gobierno y los sujetos que materializan la producción agroindustrial.

Un argumento más, acerca de la rentabilidad y la sostenibilidad viene de parte de Méndez, Mier \& Giménez, (2020):

Durante el trabajo de campo se realizaron entrevistas en plantaciones, localidades rurales y en la ciudad de Palenque. Los hallazgos indican que, en 
la zona recorrida, se está gestando una territorialidad expresada en la dependencia de los campesinos-palmeros a los ingresos monetarios de la venta del fruto de la palma, quienes en su mayoría no obtienen ganancias económicas considerables, pero sí las mínimas necesarias para mantenerse en la cadena de producción. (p. 1). La tendencia es clara: la expansión del modelo agroindustrial de producción del aceite de palma predomina en Palenque, territorio que está ubicado al norte de Chiapas y colinda con el estado de Tabasco.

Otra manifestación de rentabilidad y sostenibilidad agroindustrial se deriva de los subproductos de la palma aceitera, tal como relata Barragán et al. (2020):

Los subproductos de la industria de palma africana, especialmente el OPDC, lograron demostrar una respuesta positiva en el incremento de la producción de leche bajo las condiciones de pequeño productor doble propósito en el sur del departamento del Atlántico. Sin embargo, se hace necesario considerar experimentos de mayor plazo para evaluar la sostenibilidad de la producción, la calidad de la leche y la viabilidad económica, social y ambiental de estos esquemas de suplementación estratégica bajo condiciones de pequeño productor doble propósito. (p. 12). Para arribar a está única conclusión, los autores evaluaron el efecto de subproductos de la palma africana en la producción y la calidad de la leche bajo condiciones de pequeño productor doble propósito en el sur del departamento del Atlántico.

Como es natural cuando las relaciones de producción entre los dueños de las empresas multimillonaria dedicadas al cultivo y agro-industrialización se vuelven hostiles, entonces aparecen los conflictos en busca de hacer prevalecer derechos conculcados, así ocurrió en el Perú, tal como relata Borasino, (2016):

En agosto de 2015, los productores demandan al Gobierno, a través de un pronunciamiento, garantizar la sostenibilidad de las 77000 hectáreas de palma aceitera en el país y que deje atrás su “inacción”. Específicamente, se solicita el cumplimiento de la Ley de Biocombustibles y su reglamento, y se exige la defensa de la producción nacional frente a la importación de biodiesel subsidiado. (p. 45). La Ley de Promoción del Mercado de Biocombustibles 
$\mathrm{N}^{\circ} 28054$, es una ley jurídica peruana que fue promulgada en el gobierno de Alejandro Toledo.

Refiriéndose a rentabilidad y sostenibilidad, conviene anotar el derrotero que ha transitado el Ecuador desde el 2007, en la búsqueda de crecimiento económico y reducción de la pobreza, Calderón, Dini \& Stumpo (2016) en términos pragmáticos dicen: “De los insumos destinados a la producción, el más importante es la palma africana, con una producción de US\$1.400 millones.” (p. 73). De manera que no sólo en África, Europa, Estados Unidos, India, China, entre otros muchos países, sino también en el Ecuador la palma aceitera es una alternativa para lidiar con la escasez.

Ahora bien, referirse a estrategias de gestión socioeconómica que hayan conllevado al desarrollo económico sostenible y rentable derivado de la agroindustria de palma aceitera, evidentemente existen y para rato. Eso sí, se trata de la gestión de las empresas transnacionales, las que representan los fines de control y poder de grandes grupos económicos sobre empresas pequeñas y medianas constituidas por consumidores y trabajadores que mayormente venden sus materias primas y su fuerza de trabajo.

Finalmente, la agroindustria de la palma aceitera no avizora sostenibilidad socialambiental, puesto que en diversas partes del territorio amazónico está relacionada con las violaciones de los Derechos Humanos. Además, los extensos monocultivos para los biocombustibles son una amenaza para la alimentación sana y deterioran la biodiversidad.

\section{En esa lógica racional, Dammert (2014) dice:}

Por otra parte, en todos los casos vemos que gran parte de las decisiones sobre el uso del suelo en la Amazonía están descentralizadas. Los procesos de descentralización no han terminado de consolidarse y existe una percepción generalizada de que en muchos gobiernos sub-nacionales prevalece la corrupción y escasas capacidades de gestión. La descentralización obedece a políticas de Estado que tienen como lógica acercar el poder de decisión a las zonas involucradas en una determinación que las afecta, o para ponerlo de otra forma, acercar el poder a los ciudadanos de a pie. En términos ambientales, en muchos casos ocurre que los poderes locales tienen especial interés en actividades como la expansión de la frontera agropecuaria, lo cual es legítimo; pero en ocasiones encontramos también que gobiernos subnacionales débiles son cooptados por intereses empresariales particulares. (p. 
66). En este discurso, subyace el problema de la tenencia privada de la tierra, de allí que a la agroindustria de la palma aceitera se vincula el despojo de tierras.

\section{REFERENCIAS BIBLIOGRÁFICAS}

Barragán, W., Mestra, L., Portilla, D., Mejía, A. \& Henríquez, R. (2020). Efecto de subproductos de palma africana en la producción y calidad de leche bovina en el sur del departamento del Atlántico, Colombia. En: Cienc. Tecnol. Agropecuaria, 21 (2): e1132 DOI: https://doi.org/10.21930/rcta.vol21_num2_art:1132

Borasino, E. (2016). La cadena de la palma aceitera en contexto. Lima: Consejo Latinoamericano de Ciencias Sociale.

Comisión Económica para América Latina y el Caribe (2019). Perspectivas de la agricultura y del desarrollo rural en las Américas: una mirada hacia América Latina y el Caribe 2019-2020. San José: Organización de las Naciones Unidas para la Agricultura y la Alimentación (FAO).

Calderón, Á., Dini, M. \& Stumpo, G. (2016). Los desafíos del Ecuador para el cambio estructural con inclusión social. Santiago: Naciones Unidas

Dammert, J. (2014). Cambio de uso de suelos por agricultura a gran escala en la Amazonía andina: el caso de la palma aceitera. Lima: Unidad de Apoyo de la Iniciativa para la Conservación en la Amazonía Andina (ICAA).

González, A. (2016). La agroindustria de la palma de aceite en América. En: Palmas, Vol. 37 No. Especial, Tomo II.

Harnecker, M. (1976). Los conceptos elementales del materialismo histórico. Madrid: Siglo Veintiuno Editores, S.A.

Leveau, R. (2018). Sustentabilidad de fincas productoras de palma aceitera (Elaeis Guineensis), en el valle del río Shanusi, Loreto. Lima: Universidad Nacional Agraria la Molina.

Linares, B., Zapata, E., Nazar, A. \& Suáres, B. (2018). Reconversión productiva a palma de aceite en el Valle del Tulijá, Chiapas, México. Impacto diferenciado por género. En: Agricultura, sociedad y desarrollo, Vol.15 No. 4 Texcoco. 2018. Versión Impresa ISSN 1870-5472

Madrigal, Á. \& Garbanzo, G. (2018). Uso de residuos agroindustriales en pre-viveros de palma aceitera (Elaeis guineensis, Arecaceae): crecimiento y absorción de 
nutrimentos. En: Cuadernos de Investigación UNED Vol.10 ํ2 Sabanilla, Montes de Oca. 2018

Méndez, J., Mier, M. \& Giménez, T. (2020). Expansión de la producción de aceite de palma en territorios campesinos, el caso de Palenque, Chiapas (1996-2018). En: Revista pueblos y fronteras digital. Vol.15 San Cristóbal de Las Casas. 2020 Naranjo, M. (2016). La política agropecuaria ecuatoriana: hacia el desarrollo territorial rural sostenible: 2015-2025. Quito: Ministerio de Agricultura, Ganaderia, Acuacultura y Pesca.

Naranjo, M. (2016). La política agropecuaria ecuatoriana: hacia el desarrollo territorial rural sostenible: 2015-2025. Quito: Ministerio de Agricultura, Ganadería, Acuacultura y Pesca.

Ministerio del Ambiente (2016). Perú 2030: la visión del Perú que queremos. Lima: Programa de las Naciones Unidas para el Desarrollo (PNUD).

Pertuz, A. \& Santamaría, A. (2014). La palmicultura colombiana: sostenibilidad económica, social y ambiental. Nariño: Facultad de Ciencias Económicas y Administrativas de la Universidad de Nariño. En: Revista Tendencias de la Facultad de Ciencias Económicas y Administrativas de la Universidad de Nariño del año 2014, Vol. XV, $\mathrm{N}^{\circ} 1$.

Quintero, L. \& Torres, C. (2019). Análisis de residuos sólidos de palma africana, como alternativa de aprovechamiento de energías renovables en el departamento del Cesar. Medellín. En: Ingenierías USBMED. Vol. 10, N¹. 2019 Medellín.

Rao, V. (2004). Sostenibilidad económica, social y ambiental de la producción de aceite de palma en Malasia. Discusión y tendencias del mercado. Kuala Lumpur: Asociación Malaya de Aceite de Palma. En: Palmas, Vol. 25 No. Especial, Tomo I.

Salinas, E., Mucho, E., Amado, E. \& Mendoza, J. (2017). Planeamiento Estratégico de la Palma Aceitera del Perú. Lima: Pontificia Universidad Católica del Perú.

Villafuerte, D. (2018), Entre la pasión y el bajo aguán: El rostro violento del neoextractivismo palmero en Centroamérica. En: Anuario de Estudios Centroamericanos. Vol.44. 2018. http://dx.doi.org/10.15517/aeca.v44i0.34008 
Villarán, F., Dávila, M., Urday, P., Sánchez, B. \& Vargas, R. (2014). El Modelo de Desarrollo Alternativo de la Región San Martín: Un estudio de caso de Desarrollo Económico Local (DEL). Lima: UNODC.

Zurita, T., Pucutay, J., Córdova, I, \& León, L. (2019). Estrategias financieras para incrementar la rentabilidad. Caso: Empresa Indema Perú S.A.C. Quito: Universidad Internacional del Ecuador. En: Revista de Investigación INNOVA del año 2019. Volumen 4, No.3.1 